\title{
Pränatal- und Präimplantationsdiagnostik: Diskriminierung von Menschen mit Behinderungen?
}

Tanja Krones

\section{Einleitung}

In kaum einem Bereich wird die Verbindung von medizinisch-ärztlichem Handeln und der Diskriminierung von Menschen mit Behinderungen so ausführlich diskutiert und auch in vielen Diskussionsbeiträgen so unzweifelhaft als gegeben angenommen, wie in der pränatalen (PND) oder präimplantativen (PID) Diagnostik.

Verschiedene Methoden der PND, wie die Fruchtwasserzelluntersuchung (Amniozentese) oder die Untersuchung von Zellen aus der Plazenta (Chorionzottenbiopsie) haben sich seit ihrer Etablierung in den 197oer Jahren zu Routineverfahren entwickelt, die von vielen Frauen während ihrer Schwangerschaft in Anspruch genommen werden. Zunächst wurde die Pränataldiagnostik nur in wenigen Fällen Paaren mit einem bekannten genetischen Risiko angeboten. Später wurde das Angebot um die sogenannte Altersindikation erweitert. Bekanntermaßen steigt mit zunehmendem Alter der Frauen die Wahrscheinlichkeit für Chromosomenfehlverteilungen, die u. a. zur Trisomie des Chromosoms 21 und damit zum Down-Syndrom des Kindes führen. Lange Zeit galt die Empfehlung, Frauen ab 35 Jahren eine invasive Pränataldiagnostik anzubieten, da zu diesem Zeitpunkt das Risiko von schweren Komplikationen der Amniozentese das Risiko, ein Kind mit Chromosomenfehlverteilungen bekommen, unterschreitet. In den letzten Jahren wurden neue Screeningmethoden, wie derTripletest oder das Ersttrimenonscreening entwickelt. Mittels frühem Ultraschall in der 9. bis 12. Schwangerschaftswoche, bei dem das so 
genannte dorsonuchale (Nacken-)Ödem des Embryos gemessen wird, gekoppelt mit der Messung verschiedener Blutwerte, können Wahrscheinlichkeiten für das Vorliegen von Chromosomenfehlverteilungen ermittelt werden. Ersttrimenonscreening und Tripletest betreffen nun potentiell alle Schwangeren unabhängig von ihrem Alter. Beide Screeningverfahren werden in Deutschland im Moment aber lediglich als individuelle Gesundheitsleistung (iGeL) angeboten. Nur durch eine invasive Pränataldiagnostik lässt sich nach einem positiven Screeningtest bestätigen oder widerlegen, dass das Kind beispielsweise ein Down-Syndrom hat.

Gemäß den Analysen der Perinataldaten ist in den letzten Jahren der Gesamtanteil an Frauen, die eine Amniozentese durchführen lassen, gesunken, obgleich der Anteil an über 35-jährigen Schwangeren weiter gestiegen ist. Die Autoren führen dies zum einen auf die vermehrte individuelle Nutzung der genannten Screeningmethoden zurück, durch welche ältere Frauen bei negativem Befund vermehrt auf eine Amniozentese verzichten würden (wobei jüngere Frauen bei positivem Screening-Befund sicher häufiger erst dazu gebracht werden, eine Amniozentese durchzuführen), zum anderen aber auch auf einen Wertewandel, eine zunehmend kritischere Haltung gegenüber pränataler Diagnostik in Deutschland. ${ }^{1}$

Nach einer positiven Diagnose der Pränataldiagnostik entscheiden sich bei Vorliegen eines Down-Syndroms jedoch bis zu 90\% der Frauen für einen Schwangerschaftsabbruch. Ein solcher Abbruch wird häufiger an der Grenze zur Lebensfähigkeit des Kindes durchgeführt und stellt für alle Beteiligten meist eine große Belastung dar. Bei den hierzulande angewendeten Methoden wird bei späteren Abbrüchen eine Geburt eingeleitet, die in den Schilderungen vieler Frauen als extreme Belastung empfunden wird. In Amerika werden spätere Abbrüche der Schwangerschaft häufig durch eine so genannte Dilatation and Evacuation (D\&E)-Methode durchgeführt. Dabei müssen die Frauen keine Entbindung durchleben, sondern der Gebärmutterhals wird, wie bei der Absaugmethode bei frühen Schwangerschaftsabbrüchen, unter Betäubung erweitert und das Kind herausgezogen.

Die sogenannte eugenische Indikation zum Schwangerschaftsabbruch bis zur 22. Woche nach Zeugung (24. Woche nach der letzten Menstruation) aufgrund einer Behinderung oder Erkrankung des Kindes wurde in der jetzt gültigen Fassung des $\mathbb{2} 218$ aus dem Strafgesetzbuch genommen. Dies geschah genau aufgrund des Vorwurfs, diese explizite gesetzliche Möglichkeit eines straffreien Schwangerschaftsabbruchs diskriminiere Menschen mit Behinderungen. Die medizinische (mütterliche) Indikation des Schwangerschaftsabbruchs gilt jedoch seitdem auch bei antizipierter Unzumutbarkeit der Fortführung der Schwangerschaft für die Mutter. In der Praxis fallen unter diese erweiterte medizinische Indikation nun auch die Schwangerschaftsabbrüche aufgrund des Ergebnisses eines pränatalen genetischen Tests. Durch diese seit

1 Hierbei stiegen die sehr niedrigen Zahlen an invasiver Diagnostik in Ostdeutschland, sanken aber dagegen in Westdeutschland von einem deutlich höheren Niveau. Vgl. Rauskolb (2000), Rauskolb/Wenzlaff (2004). Vgl. auch die Richtlinien zur Pränataldiagnostik der Bundesärztekammer BÄK (1998) und die Richtlinien der Deutschen Gesellschaft für Gynäkologie und Geburtshilfe zur Ultraschalldiagnostik (AWMF-Leitlinie 015/044). 
1995 geltende Regelung sind letztlich Schwangerschaftsabbrüche aufgrund fetaler Erkrankungen oder Behinderungen bis zur Geburt möglich, was von vielen Seiten, unter anderem auch von der Deutschen Gesellschaft für Humangenetik und der Deutschen Gesellschaft für Gynäkologie und Geburtshilfe als eine problematische Situation angesehen wird.

In den letzten zehn Jahren gab es hinsichtlich der Gesamtzahl der Schwangerschaftsabbrüche und hinsichtlich deren Art nach Angaben der Gesundheitsberichterstattung des Bundes kaum Veränderungen. Die Zahlen sind eher rückläufig. In 2005 wurden insgesamt 124.023 Schwangerschaften abgebrochen, davon waren 120.825 Schwangerschaftsabbrüche, die unter die Beratungsregelung fielen, 3.177 Schwangerschaftsabbrüche wurden aufgrund einer medizinischen Indikation durchgeführt, 2.049 fanden zwischen der 13 . und 23. Woche statt, und 171 waren Spätabbrüche nach der 23. Woche. Allerdings geht die Deutsche Gesellschaft für Gynäkologie und Geburtshilfe hierbei auch von einer höheren Dunkelziffer aus, da vermutlich einige Spätabbrüche als Totgeburten klassifiziert würden. ${ }^{2}$

Die PID wurde in England Ende der 1980er Jahre als mögliche Alternative zur Pränataldiagnostik, wiederum zunächst für Paare mit einem bekannten genetischen Risiko, entwickelt. Hierbei müssen sich die Frauen und Paare einer künstlichen Befruchtung (In-vitro-Fertilisation, IVF) unterziehen. Einzelne Zellen des mehrzelligen Embryos werden mittels molekulargenetischer Methoden auf die in der Familie bekannte krankheitsverursachende Genmutation untersucht, und nur ein bis drei Embryonen, welche die zur Erkrankung führende Genmutation nicht tragen, werden in die Gebärmutter eingesetzt. Auch hier kam es in den letzten Jahren zur Ausweitung des Indikationsspektrums. Lediglich $40 \%$ der PID-Untersuchungen werden mittlerweile aufgrund einer elterlichen Disposition für die Vererbung genetisch mitbedingter Erkrankungen oder Behinderungen durchgeführt. Diese Paare sind in aller Regel nicht unfruchtbar, sondern könnten ohne assistierte Reproduktion ein Kind bekommen. Sie wählen die PID, weil sie von dem Risiko für die genetische Erkrankung aufgrund vorheriger Schwangerschaften oder aufgrund einer eigenen Behinderung oder Erkrankung wissen, ein weiteres leibliches Kind wünschen und die Geburt eines Kindes mit der familiären Erkrankung sowie einen möglichen späten Schwangerschaftsabbruch vermeiden wollen. Weitere $58 \%$ der durchgeführten präimplantativen Untersuchungen an in vitro gezeugten Embryonen entfallen nach den neuesten Daten des europäischen PID- (engl. PGD-) Konsortiums ${ }^{3}$ auf das sogenannte Aneuploidiescreening (PGS). Hierbei wird die PID in der IVF bei unfruchtbaren Paaren durchgeführt, um eine Chromosomenfehlverteilung auszuschließen. Hierdurch soll die Schwangerschaftsrate in der IVF bei Paaren mit wiederholten IVF-Versuchen zu erhöhen sein; die Daten sind hierzu jedoch nicht eindeutig. In $2 \%$ der Fälle wurde die PID weltweit in Familien durchgeführt, in denen schon mehrere Kinder eines Geschlechts geboren wurden. Bei diesem sogenannten social sexing werden Embryonen gezielt aufgrund ihres Geschlechts ausgewählt, ohne dass eine geschlechtsge-

2 Positionspapier der DGGG (2003): Schwangerschaftsabbruch nach Pränataldiagnostik.

3 Vgl. Sermon et al. (2007). 
bundene Erkrankung vorliegt. In einzelnen Fällen wurde die PID in Familien angewendet, in denen ein Geschwisterkind an einer Erkrankung leidet, die durch Transplantation von Stammzellen aus dem Nabelvenenblut eines HLAkompatiblen (d.h. gewebskompatiblen) Geschwisterkindes geheilt werden könnte. Hierzu wird eine PID durchgeführt, um einen HLA-kompatiblen Spender, der zudem nicht selbst diese Erkrankung geerbt hat, zu finden.

Beide Praktiken, PND und PID, werden nun - insbesondere, aber nicht nur in Deutschland von vielen Akteurinnen und Akteuren aus verschiedenen gesellschaftspolitischen Strömungen und mit unterschiedlichen Argumenten mit einer Diskriminierung von Menschen mit Behinderungen in Zusammenhang gebracht.

Wird von einer Diskriminierung gesprochen, so stellt sich dabei nicht nur grundsätzlich die Frage, was Diskriminierung ist und wer auf welche Weise diskriminiert wird, wer also Opfer der diskriminierenden Praktik ist, sondern auch, wer wie zum ,Täter` wird. All diese Fragen werden, wie ich im Weiteren darstelle, nicht eindeutig beantwortet. Häufig wurde die Biomedizin und die von ihr beeinflusste Gesellschaft per se als Verursacher einer Diskriminierung von erwachsenen Menschen mit Behinderungen durch die PND genannt. Durch den biomedizinischen Blick auf Behinderung und auf den Embryo oder Fötus mit einer Anlage zu einer Erkrankung oder Behinderung, nach dem mit immer diffizileren Methoden ,gefahndet' und der daraufhin abgetrieben wird, würde dieser - und mit ihm stellvertretend alle lebenden Menschen mit Behinderungen - als defizitär (oder gar ,lebensunwert') klassifiziert. Zudem wurden seit den 1980er Jahren in der deutschen Debatte auch die Frauen, die eine Pränataldiagnostik in Anspruch nehmen, als Mittäterinnen klassifiziert, deren Handlungen als moralisch unzulässig anzusehen seien. Dies wurde nicht nur von Lebensschützern fundamental-christlicher Provenienz, sondern auch von Vertretern der Behindertenbewegung und aus Kreisen der feministischen Wissenschaft geäußert. So fragte Christian Judith, ein prominenter Vertreter der Behindertenbewegung ${ }^{4}$ in einem Streitgespräch eine Frau mit Parkinsonsyndrom, die der embryonalen Stammzellforschung positiv gegenüberstand: „Hättest Du mich abgetrieben?“ Zitiert seien dazu zwei weitere Beispiele unter vielen:

„Die mit der Anwendung der PID oder PND verbundenen Werturteile über Behinderungen stellen eine Diskriminierung derjenigen Menschen dar, die bereits mit denselben Merkmalen leben. Die genetischen Testverfahren sind Ausdruck der bestehenden Diskriminierung von Menschen mit Behinderungen und der in diesem Zusammenhang existierenden diskriminierenden Einstellungen und Haltungen. ${ }^{\text {"5 }}$

„Für eine Schwangerschaft, die wissentlich-willentlich mit dem Ziel herbeigeführt wird, sie abzubrechen, wenn die Pränataldiagnostik einen Krankheitsbefund ergibt, gilt das gleiche wie für die Präimplantationsdiagnostik: diese Haltung und das entsprechende Handeln ist ethisch unzulässig."

4 Vgl. Judith (2001).

5 Volz (2003), S. 84.

6 So die feministische Moraltheologin Hille Haker in einer Anhörung der Enquete-Kommission Recht und Ethik der Modernen Medizin des Deutschen Bundestages am 13.11.2000. 
Zunächst stelle ich kurz die historischen Entwicklungen der bundesdeutschen Debatte dar, die zu dieser Ansicht (und über diese hinaus) geführt haben. Im nächsten Teil schildere ich die wesentlichen Argumente, die vorgebracht werden, um eine Diskriminierung von Menschen mit Behinderungen im Kontext der Pränatal- und Präimplantationsdiagnostik zu belegen. Im letzten $\mathrm{Ab}$ schnitt fasse ich zentrale Ergebnisse unserer Untersuchungen zusammen, die wir in den vergangenen Jahren in der AG Bioethik-Klinische Ethik am Zentrum für Konfliktforschung an der Universität Marburg zur Pränatal- und Präimplantationsdiagostik durchgeführt haben. Im Lichte dieser Ergebnisse sowie der aktuellen bundesdeutschen und internationalen Debatte wende ich mich abschließend der Frage zu, ob und inwieweit der Vorwurf, Pränatal- und Präimplantationsdiagnostik implizierten eine Diskriminierung von Menschen mit Behinderungen, gerechtfertigt erscheint.

\section{Historische Wurzeln der PND- und PID-Debatte}

Die Wurzeln der bioethischen Diskussion um die Bewertung der PND und PID liegen in den Anfängen der bioethischen Debatte in Deutschland überhaupt, die mit einiger Verspätung zu anglo-amerikanischen Entwicklungen erst Mitte der 1980er Jahre begonnen wurde. ${ }^{7}$ Allerdings waren die 196oer und 1970er Jahre nicht frei von Diskussionen um bioethische Themen. Diese wurden lediglich in Deutschland nicht als solche eingeordnet, sondern als medizinischgesellschaftspolitische Problemfelder wahrgenommen, und eher von Sozialwissenschaftlern und Medizinern als von Theologen, Philosophen und/oder Medizinethikern bearbeitet. Eine erste heftige Auseinandersetzung entbrannte Anfang der 197oer Jahre um die Zulässigkeit des Schwangerschaftsabbruchs. Die feministische Debatte erreichte in Deutschland mit dem Bekenntnis „Ich habe abgetrieben“ auf der Titelseite des Stern vom 2. Juni 1971 erstmals die Mitte der Gesellschaft. In diesem Diskurs verwahrten sich Feministinnen, gemeinsam mit Politikern des liberalen und linken Spektrums, gegen jedwede moralische Verurteilung und Bevormundung von Frauen, die einen Schwangerschaftsabbruch vornehmen.

Mit der Ausweitung der Pränataldiagnostik (PND) seit Ende der 1970er Jahre durch ein immer breiteres Angebot und eine deutlich steigenden Nachfrage kam es innerhalb der feministischen Debatte um den Schwangerschaftsabbruch zu einer Kontroverse, in der sich erstmals Frauen mit Behinderungen zu Wort meldeten. Die Austragung dieser Debatte fand initial in außerparlamentarischen, dann mit der Etablierung der Partei Die Grünen auch in innerparlamentarischen neuen sozialen Bewegungen statt, die von Beginn an sowohl eng mit dem feministischen Diskurs, der Antidiskriminierungsbewegung als auch mit gentechnikkritischen Kreisen verbunden waren. Viele Sozialwissenschaftler, unter anderem Elisabeth Beck-Gernsheim und Eva Schindele, haben dieser Debatte wichtige Impulse gegeben. In den ersten Stellungnahmen aus der Sicht von behinderten Feministinnen ${ }^{8}$ wurde die Abtreibung aus sozialer

7 Vgl. zu den folgenden historischen Ausführungen auch Düwell/Steigleder (2003), Kuhlmann (2001), Gerhardt (2001), Waldschmidt (2003).

8 Vgl. Ewinkel (1985), Köbsell (1989), Degener/Köbsell (1992). 
Indikation verteidigt, die Abtreibung aufgrund der damals noch im $\mathbb{} 218$ enthaltenen embryopathischen Indikation jedoch scharf kritisiert. Dabei wurden Parallelen zwischen der Pränataldiagnostik und der alten Eugenik, der Bevölkerungspolitik seit dem 19. Jahrhundert gezogen, die in dem tausendfachen Mord von Menschen mit Behinderungen während der NS-Zeit ihren grausamen Höhepunkt erreichte. Zudem wurde in diesem Diskurs die Sicht vieler Mediziner auf Behinderungen ausschließlich als Belastung (der Gesellschaft) und defizitärer Zustand, als herrschende Moral der ,Nicht-Behinderten' kritisiert. Die These „Behindert ist man nicht, behindert wird man“, die soziale Bedingtheit und Konstruktion von Behinderung wurde dagegen gestellt. In der so genannten Singer-Debatte, aus der die Antibioethikbewegung in Deutschland Ende der $1980 e r$ Jahre entstand, ${ }^{9}$ wurden sowohl ,die Biomedizin' und ,die Humangenetik' als auch ,die Bioethik' nicht nur im Licht alter Eugenik interpretiert, sondern zusätzlich und zunehmend nach der Theorie der Biomacht des französischen Philosophen und ,Sozialarchäologen“ Michel Foucault bewertet, die dieser in seinen Frühschriften entwickelt hatte. Dieser theoretische Überbau ist bis heute in der Beurteilung der PND und PID von größter Bedeutung. Die vielen Frauen, welche die PND nutzen und sich dann im Wissen um eine Behinderung oder Erkrankung des Fötus die Frage nach Fortführung der Schwangerschaft oder Abtreibung stellen, sowie die steigende Anzahl von Paaren, die sich zur Durchführung der PID an Zentren im Ausland wenden, betreiben nach dieser These eine neue ,Alltagseugenik‘. Die Entscheidung für oder gegen die Austragung eines Kindes mit einer Behinderung oder chronischen Erkrankung wird zwar (meist, aber durchaus nicht immer, siehe oben) als Entscheidung der Frau akzeptiert; deren Autonomie wird jedoch grundsätzlich in Frage gestellt. Dabei wird unterstellt, dass eine Frau, die sich zur PND oder PID entschließt oder einen Schwangerschaftsabbruch aufgrund des Ergebnisses eines genetischen Tests durchführen lässt, einem Entscheidungsdruck seitens der (unmoralischen) Ärzteschaft und Gesellschaft unterliege, ein solches Kind nicht zu bekommen. Oder es wird behauptet, dass die Frauen, die diese Technik nutzen, die ethisch fragwürdigen gesellschaftlichen Normierungen von Gesundheit und Krankheit verinnerlicht hätten. Sie handelten daher unbewusst unmoralisch und betrieben eine ,Eugenik von unten'. Untermauert wurde diese fundamentale Kritik an Gen- und Reproduktionstechnologien durch einfühlsame biographische Studien aus den 1980er und 1990er Jahren, u. a. von Eva Schindele (1995). In diesen Studien wird von Frauen berichtet, die meist ohne ein bekanntes genetisches Risiko aufgrund der sogenannten Altersindikation, schlecht informiert in die Mühlen von invasiver pränataler Diagnostik gerieten oder sich selbstverständlich dort hineinbegeben haben. Dazu kamen (und kommen bis heute) Berichte von Eltern mit behinderten Kindern, denen gesagt wird, dass man die Geburt des behinderten Kindes mittels PND doch hätte verhindern können. Es bestehe,

9 Als zentrales Werk der Antibioethik auch im Hinblick auf ihre Geschichte siehe Braun (2000), zur Antibioethik- und Anti-Singer-Debatte siehe auch Schöne-Seifert/Rippe (1991), Boshammer et al. (1998), Düwell/Steigleder (2003), Kuhlmann (2001). 
so diese These daher insgesamt ein starker sozialer Druck, die PND anzuwenden und ein Kind mit einer Behinderung nicht zu bekommen.

Die Debatten der 1980er Jahre führten unter anderem zur Neuregelung des Schwangerschaftsabbruchs Mitte der 1990er Jahre und zur Formulierung des Embryonenschutzgesetzes 1990. Aufgrund des Embryonenschutzgesetzes ist die PID nach geltender juristischer Mehrheitsmeinung in Deutschland implizit verboten und wird bisher nicht durchgeführt.

Der jüngste bioethische Diskurs insgesamt und auch in Bezug auf Gentechnologien und Pränataldiagnostik wurde mit dem Diskussionsentwurf der Bundesärztekammer zur PID im Mai 2000 angestoßen. Dabei empfahl die Bundesärztekammer in einem mittlerweile zurückgezogenen Papier, die PID für schwere genetische Erkrankungen zuzulassen. ${ }^{10}$ Seitdem haben sich wieder viele in der Debatte zu Wort gemeldet. Sowohl die Enquetekommission Recht und Ethik der Modernen Medizin des Deutschen Bundestages als auch der 2001 gegründete Nationale Ethikrat haben zur PND und PID Stellungnahmen verfasst, in denen die Argumente der Diskriminierung von Menschen mit Behinderungen durch PND und PID eine ganz zentrale Rolle einnahmen, sowohl beim eindeutig ablehnenden Votum der Enquetekommission zur Einführung der PID als auch beim mehrheitlich positiven Votum des Nationalen Ethikrates. Auch haben sich viele medizinische Fachgesellschaften zur PND und PID geäußert. Viele Akteure aus den Anfängen der deutschen Bioethikdebatte der 1980er Jahre fungierten dabei als hinzugezogene Experten. Zudem entstand in den letzten Jahren eine „kritische Bioethik“, zu der sich v.a. „,kritische Ärzte und Wissenschaftler, bekennende Christen, Behinderte und deren Verbandsvertreter sowie Feministinnen " zählen. ${ }^{11}$ Hierbei wird in jüngster Zeit keine fundamental-oppositionelle Haltung mehr gegenüber der Bioethik/Biomedizin eingenommen. Der Diskriminierungsvorwurf wird differenziert auf der persönlichen, gesellschaftlichen und kulturellen Ebene diskutiert. So sei die Einstellung von Behindertenverbänden gegenüber Biomedizin, Bioethik und PND nicht ausschließlich ablehnend, sondern ambivalent (bis ablehnend). ${ }^{12}$ Anne Waldschmidt konstatiert zunehmend „neue Differenzen“. ${ }^{13}$ So hätten behinderte Feministinnen bemerkt, dass es auf der öffentlichen gesellschaftlichen Ebene zu einer größeren Toleranz gegenüber Menschen mit Behinderungen gekommen sei. Der eigentliche Konflikt würde jedoch an die Frauen weitergegeben. Diese würden, ungeachtet der öffentlich geäußerten Toleranz, auf der persönlichen Ebene der Nahbeziehung durch die Nutzung der PND mit nachfolgendem Abbruch der Schwangerschaft gegen die Toleranz gegenüber Menschen mit Behinderungen verstoßen.

Seit den Anfängen der bundesdeutschen Diskussion, von den $1980 e r$ Jahren bis heute, wurden Frauen und Paaren, die die PND oder PID nutzen, mögliche moralisch veritable Haltungen und Motive aufgrund der starken gesellschaftspolitischen Dominanz des Diskriminierungsvorwurfs äußerst selten

\footnotetext{
10 Bundesärztekammer (BÄK) (2000).

11 Graumann (2003a), S. 20.

12 Graumann (2006).

13 Waldschmidt (2003), S. $104 \mathrm{ff}$.
} 
attestiert. Maximal wird betroffenen Paaren ein realer Notstand (bei PND) oder ,nur' antizipierter Notstand (bei PID) zugestanden, welcher durch moralisch weitaus bessere Alternativen (Verzicht auf Kinder, Adoption oder auch: Verzicht auf PND und PID und die ,unbedingte Annahme“ eines behinderten oder kranken Kindes) gelöst werden könne. Die betroffenen Paare kamen in den wissenschaftlichen und politischen Debatten in Deutschland selbst kaum zu Wort.

\section{Bioethisch-biopolitische Argumentationsfiguren}

Im Wesentlichen liegen den facettenreichen Argumenten zum Zusammenhang von PND und PID mit einer Diskriminierung von Menschen mit Behinderungen zwei Denkfiguren zugrunde: erstens das sogenannte expressivist argument und zweitens Dammbruchszenarien auf verschiedenen Ebenen, die ich im Folgenden kurz umreiße.

\subsection{Das „expressivist argument“}

Das expressivist argument wurde erstmals von angloamerikanischen Wissenschaftlerinnen und Aktivisten aus der Disability-Rights-Bewegung Ende der 1980er Jahre formuliert. ${ }^{14}$ Demnach sendet die Praktik der PND die Botschaft an Menschen mit Behinderungen aus, dass diese es nicht wert seien, geboren worden zu sein. Hierbei sei die selektive Abtreibung im Gegensatz zur Abtreibung aus sozialen oder anderen Gründen deswegen verwerflich, da die Entscheidung gegen einen bestimmten Fötus getroffen würde, im Gegensatz zu jedem Fötus bei Frauen, die kein Kind bekommen möchten. Nicht die Abtreibung an sich wird verurteilt, sondern der Abbruch aufgrund des bestimmten Merkmals „Behinderung“, „Fehlbildung“ oder „chronische Krankheit“. Hierdurch wird der Schwangerschaftsabbruch an sich verteidigt, die selektive Abtreibung jedoch aufgrund der ausgesendeten Botschaft an die lebenden Menschen mit Behinderungen verurteilt. Wer genau die Botschaft aussendet, ob dies die Paare sind, die PND in Anspruch nehmen, oder medizinische und gesellschaftliche Institutionen sind, wird, wie oben erwähnt, nicht ganz klar.

In Deutschland hat sich insbesondere Sigrid Graumann ausführlich mit diesem Gedanken im Rahmen der Anerkennungstheorien von Axel Honneth, Nancy Fraser und Charles Taylor befasst. ${ }^{15}$

Sie argumentiert ebenfalls, wenn auch nur implizit, mit dem expressivist argument als Botschaft an Menschen mit Behinderungen und diskutiert die negative Botschaft durch die Praktik der PND und PID im Hinblick auf Formen der Anerkennung und Entwicklungen personaler Identität. Eine vollständige und unbeschädigte personale Identität entsteht demnach durch Liebe in persönlichen Nahbeziehungen und die Erfahrung von gesellschaftlicher Anerkennung eigener Rechte sowie der kulturellen Wertschätzung der eigenen besonderen Eigenschaften und Fähigkeiten. Hierdurch erwerben Kinder im

$14 \mathrm{Vgl.} \mathrm{Asch} \mathrm{(1988),} \mathrm{Wendell} \mathrm{(1996).}$

15 Vgl. Graumann (2003 b), (2006). 
Laufe ihrer Entwicklung Selbstvertrauen, Selbstachtung und Selbstwertgefühl als Formen des Selbstbezugs, ohne die eine glückliche, sichere Identitätsbildung nicht möglich ist. Nach Sigrid Graumann liegt das zentrale Problem der PND nicht auf der gesellschaftlichen Ebene der Anerkennung eigener Rechte und der Selbstachtung, die, so sieht dies auch Graumann, für Menschen mit Behinderungen weitgehend erstritten wurden. Das Defizit offenbart sich vielmehr auf der Ebene der persönlichen Nahbeziehung, dort, wo es um die kulturelle Norm der, so Graumann, unbedingten Annahme eines Kindes ginge. Genau diese Norm, dieser Anspruch auf unbedingte fürsorgliche Annahme, sei durch die Möglichkeiten der PND und PID brüchig geworden. Im Sinne eines Dammbruchargumentes könne diese Entwicklung für die Verlässlichkeit von Beziehungen überhaupt ungeahnte Folgen haben. Zudem sei neben der Annahme von Menschen mit Behinderungen die Botschaft der PND auf der kulturellen Wertschätzungsebene fatal. Hier trage die PND dazu bei, ein Bild des Menschen zu entwerfen, welcher eben nicht in seinen besonderen Fähigkeiten und Bedürftigkeiten ernstgenommen würde. Vielmehr würden kulturelle Wertemuster befördert und damit eine Botschaft ausgesandt, die das Selbstwertgefühl von Menschen mit Behinderungen zutiefst verletzten. Durch den ausschließlichen Fokus auf die Anerkennung von individuellen Rechten im bioethischen Diskurs und die Vernachlässigung der persönlichen und kulturellen Ebene seien die individual- und sozialethischen Anliegen der Behindertenbewegung von der Biomedizin und der Bioethik in wesentlichen Punkten nicht verstanden worden. Im Gegensatz zu Honneth sieht Graumann zudem durchaus einen berechtigten Anspruch auf Anerkennung der Bedürftigkeit auch in persönlichen Nahbeziehungen. Was daraus konkret für die Praktik der PND und die Reproduktionsentscheidungen von betroffenen Menschen folgt, wie solche Ansprüche an persönliche Nahbeziehungen und Fortpflanzungsentscheidungen moralisch gerechtfertigt durchgesetzt werden können und sollten, wird nicht weiter ausgeführt.

\subsection{Dammbruchszenarien}

Der zweite in der Bioethik insgesamt oft verwendete Gedankengang, der sich im Wesentlichen auf die Argumentation des deutschstämmigen jüdischen Philosophen Hans Jonas stützt, die er in seinem Buch „Das Prinzip Verantwortung“ entwickelt hat, sind so genannte sozialethische Dammbruchargumente. Durch die Zulassung einer bestimmten Praktik kommt es demnach zu einem irreversiblen Schaden auf bis zu drei verschiedenen Ebenen: ${ }^{16}$

- ein primärer (direkter, mittels jeder, als unmoralisch verstandener Einzelhandlung zugefügter) Schaden durch den Gebrauch der Praktiken. Aufgrund der in Deutschland vorgenommenen universalistischen oder pragmatischen Zuschreibung eines personalen Status an den Embryo vom Zeitpunkt der Befruchtung an kommt es zur Tötung eines Menschenwürdeträgers durch Nicht-Implantation bei IVF oder bei Abbruch einer

16 Vgl. Jonas (1979), Schöne-Seifert (1996). 
Schwangerschaft, und zudem zu einer direkten Diskriminierung, einer Verletzung des Selbstwertgefühls von Menschen mit Behinderungen, deren kulturelle Gleichwertigkeit durch die Praxis in Frage gestellt wird.

- ein sekundärer Schaden, da die Praxis missbraucht werden könnte (hier: PID/PND zur Geschlechtsselektion aus Präferenzgründen oder aus unbegründeter Abwehr von Menschen mit Behinderungen).

- ein tertiärer Schaden, indem die Gesellschaft durch die in Frage stehenden Praktiken hin zu unmoralischeren Haltungen sozialisiert wird. Verwiesen wird insbesondere auf die Entstehung eines sozialen Drucks auf Frauen, PND und PID gegen ihren Willen anzuwenden, sowie auf eine mittelbar stärkere Diskriminierung behinderter Menschen in der Gesellschaft, deren Geburt durch PND/PID als verhinderbar erfahren wird.

Sowohl bei der Annahme eines sekundären als auch eines tertiären Schadens greift die Argumentation meist, wie oben geschildert, auf die Theorie der Biomacht von Foucault zurück. ${ }^{17}$ Während bei der Annahme eines tertiären Schadens die Frauen selbst nicht zu Mittäterinnen werden (müssen), führt die zusätzliche Annahme eines primären und sekundären Schadens zur Verurteilung derjenigen Frauen, die diese Praktiken durchführen, als unmoralisch handelnde Akteurinnen, gegen die der nun primäre Hauptakteur Embryo zu schützen ist. Lediglich eine Ausnahme wird in dieser Argumentationsfigur noch als ethisch zulässig erachtet: Die Entscheidung einer Frau zum Schwangerschaftsabbruch, wenn sie aufgrund eines jedweden, ihr selbst überlassenen Motivs generell oder zu einem bestimmten Zeitpunkt kein Kind bekommen möchte, da Frauen ansonsten einem Gebärzwang unterlägen, der auch aus feministischer Sicht unhaltbar sei. Die Schwangerschaft wird als gemeinsame körperlich verbundene Zeitspanne zweier Menschenwürdeträger, der Abbruch als Selbstverteidigung analog zur Tötung in Notwehrsituationen konstruiert. Das Motiv, einen Fötus aufgrund einer vorgeburtlich diagnostizierten Erkrankung oder Behinderung abzutreiben, oder bei der IVF nur die Embryonen einzusetzen, die eine durch PID diagnostizierte Erkrankung oder Chromosomenfehlverteilung nicht aufweisen, wird dagegen aus unterschiedlichen Argumentationen heraus zu einer unmoralischen Handlung erklärt. Die Entscheidung für oder gegen die Austragung eines Kindes mit einer Behinderung oder chronischen Erkrankung wird in der Argumentation, wie oben geschildert, zwar meist als Entscheidung der Frau akzeptiert, deren Autonomie jedoch grundsätzlich in Frage gestellt. Ähnlich wird auch die Entscheidung von sterilen Frauen (oder Frauen mit einem sterilen Partner) für die Inanspruchnahme einer IVF als nachvollziehbar, der zugrunde liegende Kinderwunsch jedoch als vorwiegend gesellschaftlich auf der Basis eines patriarchalischen Frauen-bzw. Mutterbildes konstruiert und damit ebenfalls als nicht wahrhaft autonom gewertet.

Unabhängig von der möglichen theoretischen Problematisierung des Biomachtsargumentes ist die empirische Überprüfung der zugrunde liegenden

17 Vgl. Foucault $(1971,1988)$. 
Annahmen notwendig. Insbesondere müssen sich Dammbruchargumente, welche nach Hans Jonas' Postulat der Heuristik der Furcht (1979) deren Verfechter mit größerer Macht ausstatten, als diejenigen, die von der Technik profitieren würden, einer - wenn auch schwierigen - empirischen Überprüfung stellen.

Hypothetisch angenommen wird in der dargestellten Argumentation,

1. dass Frauen aufgrund eines externalen gesellschaftlichen Drucks (der Foucault'schen ,alten“ Souveränitätsmacht), ein Kind mit einer Behinderung nicht zu bekommen, PND und PID gegen ihren Willen anwenden,

2. dass Frauen gemäß der Theorie der Biomacht in der Verbindung von Technologien der Souveränitätsmacht und des Selbst aus eigenen internalisierten Abwehrhaltungen gegenüber Menschen mit Behinderungen durch PND und PID die Geburt eines behinderten Kindes verhindern wollen,

3. dass sich die Bedingungen von Menschen mit Behinderungen verschlechtern werden, wenn die PND sich weiter ausbreitet und die PID zugelassen wird, da die Geburt von Kindern mit Behinderungen als verhinderbar erfahren wird und

4. dass Menschen mit Behinderungen sich direkt und zunehmend durch die Anwendung und Ausweitung der PND und des Schwangerschaftsabbruchs diskriminiert fühlen.

Wie steht es aber empirisch um diese Zusammenhänge? Und: Inwieweit sind die bisher vorgebrachten Argumente, die PND und PID klar als diskriminierende Praktiken ausweisen und wesentlich die Biopolitik in Deutschland bestimmt haben, gut begründet?

\section{Lebensweltliche Ansichten und internationale Diskurse}

Anders als in vielen unserer europäischen Nachbarländer sind diejenigen, die am unmittelbarsten betroffen sind, in den Experten- und Parlamentsdebatten kaum gehört worden: Paare, die aufgrund einer genetisch vererbbaren Erkrankung und bei bestehendem Kinderwunsch ,wissentlich-willentlich“ eine Schwangerschaft eingehen, bei der die Wahrscheinlichkeit groß ist, dass das zukünftige Kind eine genetisch mitbedingte Erkrankung erbt. Aber nicht nur diese so genannten Hochrisikopaare, sondern auch die Bevölkerung wird bisher nicht in biopolitische Entscheidungen einbezogen. Während beispielsweise in England die für die Zulassung der PID zuständige Behörde, die Human Fertilisation and Embryology Authority, Befragungen durchführt und Diskussionsforen veranstaltet, deren Ergebnisse einen direkten Einfluss auf die Politik ausüben, werden in Deutschland solche, deliberative polls ' bisher nicht in Anspruch genommen. Auf die Hintergründe, die meiner Ansicht nach zu diesen - aus kontextsensitiver wie demokratietheoretischer Sicht - hochproblematischen biopolitischen Entscheidungsprozessen in Deutschland führen, bin ich an anderer Stelle ein- 
gegangen ${ }^{18}$ Bioethische Diskurse haben die Neigung, einen starken nationalen Bias zu haben. Erkenntnistheoretisch weiß die heutige Wissenschaftstheorie generell um viele blinde Flecke, um vermeintlich universell fraglos gegebene Wahrheiten, deren gesellschaftlich historische Bedingtheit erst dann teilweise offenbar wird, wenn man einen ethnographischen oder historischen Blick, also eine Außenperspektive auf die eigene Gesellschaft einnimmt und sich andererseits induktiv qualitativ mit dem realen lebensweltlichen Handeln und Denken befasst, statt sich allein in Expertendiskursen zu bewegen.

Wir haben in unseren empirisch-bioethischen Studien in der interdisziplinären Arbeitsgruppe Bioethik-Klinische Ethik am Zentrum für Konfliktforschung versucht, solche Perspektiven zu eröffnen. Bezogen auf die oben genannten Zusammenhänge ergaben unsere qualitativ wie quantitativ repräsentativ angelegten Untersuchungen folgende Befunde: ${ }^{19}$

\subsection{Ambivalenz statt Eindeutigkeit ...}

In der Debatte sind - sowohl von Gegnern als auch von Befürwortern der PND und PID - viele Schwarz-Weiß-Bilder gemalt und Lager gebildet worden, die den realen lebensweltlichen Konflikten wenig entsprechen. So wurde von der einen Seite komplett negiert, dass Behinderungen für Betroffene, ihre Eltern und Geschwister eine sehr große Belastung darstellen können, andererseits wurde das „Leiden an Behinderung“ als fraglos gegeben angenommen. Weder ist Behinderung allein ein soziales Konstrukt, noch bedeutet eine Behinderung unbedingt subjektives Leid. Die Übergänge zwischen Behinderung und Erkrankung sind fließend. Hierbei ist es nicht so, dass Ärzte (,die Biomedizin 'oder ,die Humangenetik') grundsätzlich immer diejenigen sind, die Krankheiten oder Behinderungen aggravieren. In unseren Befragungen von Paaren, die selbst (bei autosomal dominantem Erbgang) oder deren Kinder eine genetisch bedingte Erkrankung oder Behinderung haben, bewerteten Ärzte den Schweregrad von Erkrankungen mit früh tödlichem Ausgang tendenziell gravierender als die betroffenen Eltern. Dagegen bewerteten Eltern von Kindern mit chronisch verlaufenden Erkrankungen, wie Mukoviszidose und verschiedene Muskelerkrankungen, diese im Durchschnitt in den quantitativ-repräsentativen Befragungen als schwerwiegender als die behandelnden Ärzte. Ein Arzt äußerte in den qualitativen Interviews beispielsweise, Mukoviszidose sei für ihn „Normalität auf anderem Niveau“. Dies sehen Eltern, deren Kind mit Mukoviszidose groß geworden ist, ähnlich, nicht aber Eltern, deren Kind an einer Komplikation der Mukoviszidose im Alter von acht Jahren gestorben ist. In einem anderen Fall berichteten Eltern von einem Kind mit einer Stoffwechselerkrankung, das als einjähriges Kind fast gestorben wäre und nun im Alter von drei Jahren gerade sitzen gelernt und erste Worte sprechen konnte, dass ihnen in der humangenetischen Beratung gesagt worden sei, in ihrem Fall würde bei einer weiteren Schwangerschaft die Durchführung einer Pränataldiagnostik und ein Schwangerschaftsabbruch

18 Krones (2006a), Krones/Richter (2004).

19 Die ausführlichen Ergebnisse finden sich in verschiedenen Veröffentlichungen, u. a. in Krones (2006 b), Krones et al. (2004), (2005), (2006). 
nicht in Frage kommen. Viele Eltern, die selbst betroffen sind, unter anderem auch ein Behindertenpädagoge und seine Frau (Lehrerin), eine Hebamme aus einem Geburtshaus mit einer genetisch mitbedingten Erkrankung, und einige Geschwisterkinder würden bei der familiär bestehenden Erkrankung eine PND durchführen lassen oder haben diese bereits durchgeführt und würden zum größten Teil auch einen Abbruch der Schwangerschaft in Erwägung ziehen. All diejenigen, die sich in ihrem privaten und/oder beruflichen Leben bereits mit größter Sorgfalt und Liebe um Menschen mit Behinderungen kümmern, oder die auch selbst betroffen sind, würden nach den geschilderten Argumentationen ihre Intoleranz gegenüber Menschen mit Behinderungen hiermit zum Ausdruck bringen und unmoralisch handeln. Außer in einem Fall hat keiner der 58 von uns qualitativ Befragten (deutsch- und türkischstämmige IVF-Paare und ,Hochrisikopaare‘, Pädiater, Hebammen, Ethiker, Gynäkologen und Humangenetiker), bis auf eine Freundin eines Vertreters einer Behindertenorganisation, Frauen moralisch für die Durchführung einer PND und die Entscheidung zum Schwangerschaftsabbruch verurteilt.

\section{2 ... auch in den Behindertenverbänden}

In den Behindertenverbänden herrscht, anders als von einigen prominenten Vertretern geäußert, über die Bewertung der PND und PID ebenfalls keineswegs Einigkeit. Vielmehr gibt es durchaus einen heftigen Streit zwischen den in den Verbänden organisierten Eltern mit einem familiären Risiko und den von (nur teils genetisch bedingten) Behinderungen betroffenen Mitgliedern, so ein Leiter eines Behindertenverbands. Die Stellungnahmen der Selbsthilfeorganisationen Mukoviszidose e. V. und auch der Deutschen Gesellschaft für Muskelkranke fallen, wie auch die Stellungnahmen verschiedener Behindertenverbände im Ausland, dementsprechend nicht so aus, dass beispielsweise die PID eindeutig abgelehnt wird. Ein selbst von einer erblichen Form einer Behinderung betroffener Vertreter eines Behindertenverbandes meinte zur Frage, wie er selbst zur PND stehen würde, hier müsse er zwischen der politischen Ebene und der privaten Entscheidung differenzieren. Auf der politischen Ebene würde er die PND und PID ablehnen, er selbst könne es sich aber praktisch, trotz seiner Helfer ehrlicherweise schwer vorstellen, wie es für ihn als behindertem Vater wäre, ein Kind mit einer Behinderung großzuziehen. Die Frage, die Christian Judith im oben zitierten Streitgespräch an eine von Parkinson betroffene Frau gestellt hat, ist daher zu einem Teil an den falschen Adressaten gerichtet. Er müsste diese ebenso an sich selbst richten, sich fragen (und ehrlich beantworten), ob er, wenn er Vater werden würde, auf keinen Fall eine PND und einen Schwangerschaftsabbruch in Erwägung ziehen würde.

Viele Paare berichteten von einer geringen Bereitschaft zu verstehen, was es tatsächlich hieße, für ein schwerstbehindertes Kind zu sorgen, und damit über eine Verharmlosung und Verklärung ihrer Situation. Genau in der Ignoranz der hohen Belastung durch ein sehr geliebtes Kind mit einer Behinderung liege ein wesentliches Problem. Eine Mutter verwendete hierbei das Bild eines schweren Rucksacks auf ihrem Rücken, mit dem man immer weiter ginge und froh sei, dass man nicht fiele, und das einzige Signal sei: „Mensch, geh doch grade“. 


\subsection{Sozialer Druck: in zwei Richtungen}

Die „kritische Bioethik“ hat sich in der Rolle der machtlosen Opponenten und Häretiker, die sich grundsätzlich gegen die Herrschenden wenden und für die richtige Sache eintreten, gut eingerichtet. Viele derjenigen, die diese Richtung maßgeblich vertreten, sitzen jedoch nicht nur bereits lange selbst in einflussreichen Machtpositionen. Diese Auffassungen, unter anderem der enge Zusammenhang von PND und PID und der Diskriminierung von Menschen mit Behinderungen haben die Gesetzgebung und die Voten der Enquetekommissionen des Bundestages zu bioethischen Fragen wesentlich beeinflusst. Frauen, die heutzutage Mütter werden, wurden durch verschiedene Frauenbilder und Ansichten zur Pränataldiagnostik geprägt. Die von feministischer und christlicher Seite, von ProfessorInnen, BioethikerInnen, Kirchenvertretern und PolitikerInnen seit den 1970er Jahren geäußerte Kritik an Reproduktionstechniken (und auch an ihren Nutzerinnen) ist selbstverständlich auch in der Gesellschaft angekommen und beeinflusst das Reproduktionsverhalten. Es besteht, so ergaben unsere qualitativen Interviews, daher für Paare, die vor (fast immer hochambivalenten) Fortpflanzungsentscheidungen stehen, ein sozialer Druck in zwei Richtungen: eine PND oder IVF/PID anzuwenden, aber auch, diese nicht anzuwenden, sowie Kinder zu bekommen und nicht zu bekommen. Paaren wird sowohl ein selektiver Schwangerschaftsabbruch nahegelegt, als auch ein solcher verweigert; für die Durchführung einer PID müssen in Deutschland lebende Paare ins Ausland fahren, was sie auch gemäß den Daten der belgischen, spanischen und niederländischen Zentren trotz großer Mühen in nicht unbeträchtlichem Maße in Anspruch nehmen. Der soziale Druck, eine PND oder PID durchzuführen, wurde von den 162 befragten Hochrisikopaaren, ebenso wie von 149 Paaren ohne bekanntes genetisches Risiko insgesamt als der am wenigsten wichtige Nachteil bewertet. In der von uns repräsentativ befragten Bevölkerung wurde die wahrgenommene Kontrolle über Fortpflanzungsentscheidungen insgesamt als hoch, und der soziale Druck, eine PND durchzuführen, bei denjenigen mit noch bestehendem Kinderwunsch (30\% von 1.027 Befragten) als gering bewertet, obgleich der Faktor sozialer Druck in dieser Richtung gemäß der empirischen Modelle auf prospektive Entscheidungen einen messbaren Einfluss hat. In der repräsentativen Untersuchung haben wir jedoch nach dem Druck, eine PND nicht anzuwenden, der in den qualitativen Interviews geäußert wurde, nicht gefragt.

\subsection{Einstellung von Nutzern der PND und PID zu Menschen mit Behinderungen}

Um eine zentrale Hypothese des Zusammenhangs von diskriminierenden Einstellungen und Reproduktionsentscheidungen zu untersuchen, haben wir in allen Stichproben die Einstellung gegenüber Menschen mit Behinderungen mittels zuvor validierter Skalen erhoben. Diejenigen, die eine PND bereits genutzt haben, und auch diejenigen, die voraussichtlich eine PID im Ausland durchführen lassen wollen (17\% der ,Hochrisikopaare“ mit Kinderwunsch, $9 \%$ der IVF-Paare) haben keine ungünstigere, sondern teilweise sogar eine günsti-

gere Einstellung gegenüber behinderten Menschen als diejenigen, die andere 
Reproduktionsentscheidungen favorisieren. Ein deutlicher Zusammenhang besteht jedoch auf der Einstellungsebene: Diejenigen, die sehr positive Einstellungen gegenüber der PND und PID haben, und letztere beispielsweise weitestgehend liberalisieren wollen, haben eher ungünstigere Einstellungen, sowohl in den Expertenstichproben als auch in den Paar- und Bevölkerungsstichproben.

Was folgt nun aus den dargestellten Ergebnissen im Hinblick auf die postulierten Zusammenhänge und die zugrundeliegenden Thesen und Argumentationsstränge?

Hinsichtlich des expressivist argument muss konstatiert werden, dass bei den Nutzerinnen und Nutzern von PND und PID nach allem, was wir bisher wissen, keine negative Einstellung gegenüber Menschen mit Behinderungen besteht. Die Entscheidung, die Techniken zu nutzen, scheint anders motiviert und durch andere Faktoren verursacht zu sein. Die Botschaft geht also nicht bewusst von den Nutzern aus, deren Haltungen mit diesem Argument so nicht als moralisch unzulässig gewertet werden könnten. Hier müssen andere Geschütze aufgefahren werden, will man die Annahme, die Motive zum und die Praktik des selektiven Schwangerschaftsabbruchs und der PID seien moralisch grundsätzlich verwerflich, aufrechterhalten. Dass dies der Fall ist, wird in Deutschland kaum in Frage gestellt. Fraglose Gegebenheiten sind jedoch aus sozialwissenschaftlicher Sicht keine Wahrheiten, sondern trügerische Klarheiten des herrschenden Diskurses. Das Unbewusste, so Emil Durkheim, ist die Geschichte. Daher lohnt es sich, auf die geäußerten tief verwurzelten, gesellschaftshistorischen Menschen- und Familienbilder zu schauen, die in der bundesdeutschen Debatte als fraglos gegeben angenommen werden. Hierbei helfen interkulturelle Vergleiche und Außenperspektiven. Hier finden wir, zumindest diejenigen, die sich nur im bundesdeutschen Diskurs bewegen, Erstaunliches. Jüngst veröffentlichte komparative Arbeiten zur bioethischen und biopolitischen Debatte um Reproduktionstechnologien in Deutschland und Israel konstatieren beispielsweise folgendes: ${ }^{20}$

In der israelischen Bioethik und Biopolitik wird die Nutzung der PND - und selbst die Auswahl eines HLA-kompatiblen Geschwisterkindes durch PID, die auch im positiven Votum des Nationalen Ethikrates als unethisch angesehen wurde - vor allem als Ausdruck der Verantwortung und Liebe von Eltern gegenüber ihren Kindern verstanden. Zukünftige Kinder und ihre Eltern werden nicht als vollständig autonome Wesen, sondern als Teil der sozialen Gruppe Familie wahrgenommen, in der Abhängigkeiten und gegenseitige Verantwortungen bestehen. Das Recht auf Kinder wird als eines der fundamentalsten Menschenrechte interpretiert, zu dessen Verwirklichung gesellschaftliche Ressourcen zur Verfügung zu stellen sind. Bei der Entscheidung für oder gegen ein Kind mit einer Behinderung spielt wesentlich auch die Fürsorge gegenüber bereits geborenen Geschwisterkindern (mit und ohne) Behinderungen eine Rolle. Eltern hätten bei der Entscheidung für eine PND nicht (nur) die Sorge und das Motiv, dass sie ein behindertes Kind nicht lieben würden, sondern dass sie dieses zu sehr lieben, um ihm und auch den Geschwistern ein Leben

20 Hashiloni-Dolev (2007). 
mit einer „geschädigten Physis“, wie Andreas Kuhlmann einmal Behinderungen bezeichnete, zuzumuten. ${ }^{21}$

In der bundesdeutschen Gesellschaft scheint, so diese Außenperspektive, bei Intellektuellen und Politikern die Vorstellung vorzuherrschen, dass ein starker Antagonismus und Interessenskonflikt zwischen Eltern und Kindern bestehe. Das Recht auf Kinder würde eher gering geschätzt, auch sei die Wichtigkeit, Kinder zu bekommen, in Deutschland laut Geburtenstatistik eher gering ausgeprägt. Die Familie werde in den Schriften vieler Intellektueller, wie zum Beispiel von Jürgen Habermas und auch in den biopolitischen Stellungnahmen als potentiell hochexploitative Gruppe, als eine Gemeinschaft von unabhängigen Menschen mit eigenen gegensätzlichen Interessen konstruiert. In Entscheidungen von Eltern werde kein Vertrauen gesetzt. Während in Israel eine ideale Elternschaft die Nutzung pränataler Diagnostik einschließe, würden in Deutschland die unbedingte Annahme des Kindes und der natürliche, schicksalhafte Verlauf von Schwangerschaft und Geburt als Ideal der elterlichen Verantwortung konstruiert.

Es gibt noch viele andere Perspektiven, welche die fraglos gegebenen Wahrheiten, Menschen- und Familienbilder im deutschen biopolitischen Diskurs in Frage stellen, so Ansichten aus der islamisch geprägten Kultur (die trotz ihres mittlerweile großen Anteils an der deutschen Kultur in den bundesdeutschen biopolitischen Gremien überhaupt keine Rolle spielt), oder auch die Untersuchungen der amerikanischen Feministin und Behindertenaktivistin Rayna Rapp, ${ }^{22}$ die Frauen, welche die PND nutzen, nicht als bewusst oder unbewusst behindertenfeindlich motivierte Menschen, sondern als moralische Pioniere sieht, in einer Zeit, da technisch gesellschaftliche Koproduktionen sowohl Zwang als auch Ermöglichung, Freiheiten und neue Abhängigkeiten schaffen. Hinsichtlich der Abtreibung eines Kindes mit Down-Syndrom finden sich bei Rayna Rapp auch aufgrund der eigenen Lebensgeschichte neue Einblicke, welche die holzschnittartigen Argumentationen, warum Frauen sich gegen ein Kind mit Down-Syndrom entscheiden, zu lebensnäheren sensibleren Bildern formen. Auch diese Ansichten haben natürlich ihre eigenen blinden Flecke. Hinsichtlich der umstandslosen Annahme, dass die Nutzung der PND und der selektive Schwangerschaftsabbruch grundsätzlich moralisch verwerflich seien, regen diese Perspektiven, so hoffe ich, zumindest zum weiteren Nachdenken und Nachlesen an. Gegen die Schlagkräftigkeit des expressivist arguments sind weitere Argumente angebracht worden, die hier nicht ausführlich geschildert werden können. ${ }^{23}$ Im Wesentlichen richtet sich die Kritik jedoch auf folgende Aspekte:

Diskriminierung ist ungerechtfertigte Ungleichbehandlung und richtet sich zudem auf die öffentliche Sphäre der Fernbeziehungen. Werden Eltern und Kinder als individuelle Personen ohne Abhängigkeiten und mit allein eigenen, gegenläufigen Interessen konstruiert, könnte das Antidiskriminierungsverbot greifen. Diese Auffassung der Eltern-Kind-Beziehung entspricht

21 Kuhlmann (2003), S. 157.

22 Vgl Rapp (2000).

23 Vgl. dazu Lindemann-Nelson (2000), Edwards (2004). 
jedoch in keiner Weise der Situation und dem Verhältnis, in dem zukünftige Eltern tatsächlich zu ihren zukünftigen Kindern stehen. ${ }^{24}$

Wenn Paare bewusst keine Kinder bekommen, oder eine Frau ihr drittes Kind oder ein Kind abtreibt, nachdem sie von ihrem Partner verlassen wurde, möchte sie keine Botschaft an alle Kinder, an dritte Kinder oder an Kinder von alleinerziehenden Müttern richten, dass ihr Leben nicht lebenswert ist. Sie glauben vielmehr, dass es für sie und ihre Familie besser ist, in ihrer Situation, mit den vorhandenen Ressourcen und Abhängigkeiten, genau dieses bestimmte Kind nicht zu bekommen. Genau so stellt sich die Entscheidung von den meisten Paaren dar, die sich gegen ein Kind mit einer Behinderung oder Erkrankung entscheiden. Wenn sie eine familiäre Erkrankung haben, wissen diese Paare in der Regel, worauf sie sich einlassen würden. Ihre Entscheidung ist daher zumindest wohlinformiert. Dies trifft jedoch sicher nicht auf viele Schwangere $z u$, die, ohne umfassend in ihrem Sinne aufgeklärt worden zu sein, durch Screeningverfahren vor diese Fragen gestellt werden. Hier besteht ohne Zweifel ein großes Defizit, welches zur hochberechtigten Kritik an der derzeitigen Praktik der Aufklärung in der Schwangerschaftsvorsorge geführt hat. Die ,Routineschwangerschaftsvorsorge“ ist daher unbedingt verbesserungswürdig. Dabei muss dem Recht auf Wissen ebensoviel Raum eingeräumt werden wie dem ebenso zentralen Recht auf Nicht-Wissen. Das eingeforderte genetische Inkognito des zukünftigen Kindes oder gar die Vorenthaltung von Informationen über Möglichkeiten der PND, wie von einer Schwangerschaftsberaterin der Diakonie im Deutschen Ärzteblatt vorgeschlagen, ${ }^{25}$ sind aus ethischer Sicht aber nicht zu rechtfertigen. Weyma Lübbe hat im Sinne dieser Überlegungen in einem ihrer Aufsätze fundiert dargelegt, warum das Diskriminierungsverbot, der Justitia-Aspekt der öffentlichen Sphäre, nicht in der Nahbeziehung gelten kann und der Caritas-Aspekt in der privaten Sphäre nicht erzwungen werden kann. Sie resümiert:

„Diejenigen, die sich von den dazu hier vorgetragenen Überlegungen nicht überzeugt sehen, und nach wie vor der Ansicht sind, dass die Präimplantationsdiagnostik ein Recht der betroffenen Embryonen missachte, von ihrer genetischen Mutter unterscheidungsfrei angenommen zu werden, mögen bitte folgendes tun: Sie mögen ein Bild der Institutionen einer Gesellschaft entwerfen, die das Diskriminierungsverbot generell - und nicht nur, diskriminierenderweise, reproduktionswilligen Frauen gegenüber - in der Privatsphäre der Bürger zur Geltung bringt. Dann könnte man darüber sprechen, ob man in einer solchen Gesellschaft leben möchte. “26

Jedoch: Die Botschaft des expressivist argument kommt an. Zwar gibt es bisher keine großangelegten Untersuchungen zu Ansichten von Menschen mit Behinderungen über die PND. Die Aussage von vielen in der Behindertenbewegung aktiven Wissenschaftlern und Betroffenen, dass sich Menschen verletzt und diskriminiert fühlen, und die Technik ihnen Angst und sie glauben mache, dass die Gesellschaft Behinderung (und damit ihre Existenz) zunehmend als

24 Vgl. Wiesemann (2006).

25 Braun (2006).

26 Lübbe (2003), S. 215. 
vermeidbar ansieht, müssen sehr ernst genommen werden. Unsere Untersuchungen ergaben zudem nicht nur, dass Nutzerinnen und Nutzer der PND und PID keine ungünstigeren Einstellungen gegenüber Menschen mit Behinderungen haben, sondern auch, dass Einstellungskonglomerate in der Gesellschaft bestehen, in denen ungünstige Einstellungen gegenüber Menschen mit Behinderungen mit sehr positiven Einstellungen gegenüber selektiven Schwangerschaftsabbrüchen und der PID verbunden sind. Dies kann durchaus im Sinne des Dammbruchsargumentes als möglicher tertiärer Schaden und als negative Botschaft der Gesellschaft an Menschen mit Behinderungen interpretiert werden. Zwar zeigen makrosoziologische Untersuchungen, beispielsweise von Wolfgang van den Daele (2003), dass Vorurteile gegenüber behinderten Kindern im Längsschnitt abgenommen haben und sich die Integration bzw. Inklusion behinderter Menschen trotz weitverbreiteter Nutzung der PND in den letzten Jahrzehnten deutlich verbessert hat. Dies heißt jedoch nicht, dass diese Vorurteile verschwunden sind - sie werden möglicherweise nur subtiler formuliert. Zum anderen besteht die Gefahr, dass durch ein immer weiter um sich greifendes ökonomisches Denken wieder weniger Ressourcen für kranke und behinderte Menschen verwendet werden. Die von uns befragten Experten waren auch zu großen Teilen der Ansicht, dass die Einführung der PID gesellschaftlich negative Folgen für Menschen mit Behinderungen haben könnte.

Es ist zwar schon häufiger gesagt worden, aber es ist meiner Ansicht nach dennoch richtig: Solchen Tendenzen muss mit politischen Mitteln beigekommen werden, nicht aber mit strafrechtlich bewehrten Verboten und mit Anschuldigungen gegenüber denjenigen, die in unserer Gesellschaft Väter und Mütter werden und ihr Leben mit Kindern teilen wollen, sich aber aus guten Gründen gegen ein Leben mit einem behinderten oder kranken Kind entscheiden. So kommen wir vielleicht in nicht allzu ferner Zukunft dahin, den Fortpflanzungsentscheidungen von zukünftigen Eltern in Deutschland wieder mehr zu trauen, Reproduktionstechniken als menschliche gesamtgesellschaftliche Produkte, die sowohl Zwang als auch Ermöglichung bedeuten können, anzusehen und Menschen mit Behinderungen eindringlicher zu vermitteln, dass von unserer Gesellschaft keine Botschaft - durch welche Praktik auch immer - ausgehen soll und darf, dass ein Leben mit Behinderungen nicht lebenswert sei.

\section{Literatur}

Asch (1988): Adrienne Asch, Reproductive Technologies and Disability, in: Sherill Cohen, Nadine Taub, Reproductive Laws for the 1990s, New York 1988, p. 69-124

Braun (2000): Kathrin Braun, Menschenwürde und Biomedizin, Frankfurt a. M. 2000

Braun (2006): Kathrin Braun, Der Wunsch nach dem perfekten Kind, Deutsches Ärzteblatt 40 (2006), S. A295-297

Bundesärztekammer (1998): Bundesärztekammer, Richtlinien zur pränatalen Diagnostik von Krankheiten und Krankheitsdispositionen, Deutsches Ärzteblatt 95 (1998), 50, S. A3236-3242

Bundesärztekammer (2000): Bundesärztekammer, Diskussionsentwurf zu einer Richtlinie zur Präimplantationsdiagnostik, Deutsches Ärzteblatt 9 (2000), S. A 461-464 
Degener/Köbsell (1992): Theresia Degener, Swantje Köbsell „Hauptsache, es ist gesund“? Weibliche Selbstbestimmung unter humangenetischer Kontrolle, Hamburg 1992

Deutsche Gesellschaft für Gynäkologie und Geburtshilfe (2003): Deutsche Gesellschaft für Gynäkologie und Geburtshilfe (DGGG), Schwangerschaftsabbruch nach Pränataldiagnostik, www.aerzteblatt.de/v4/plus/ down.asp?typ=PDF\&id= 1136

Düwell/Steigleder (2003): Marcus Düwell, Klaus Steigleder, Bioethik - Zu Geschichte, Bedeutung und Aufgaben, in: Marcus Düwell, Klaus Steigleder (Hrsg.), Bioethik. Eine Einführung, Frankfurt a. M. 2003, S. 12-40

Edwards (2004): Steve D. Edwards, Disability, identity and the „expressivist objection“, Journal of Medical Ethics 30 (2004), p. 418-420

Ewinkel et al. (1985): Carola Ewinkel et al. (Hrsg.), Geschlecht: behindert - Besonderes Merkmal: Frau. Ein Buch von behinderten Frauen, München 1985

Foucault (1971): Michel Foucault, Die Ordnung des Diskurses, Frankfurt a. M. 1971/1977

Foucault (1988): Michel Foucault, Die Geburt der Klinik. Eine Archäologie des ärztlichen Blicks, Frankfurt a. M. 1988

Gerhardt (2001): Volker Gerhardt, Der Mensch wird geboren. Kleine Apologie der Humanität, München 2001

Graumann (2003 a): Sigrid Graumann, Bioethik oder Biopolitik? Die öffentliche Debatte über die "Selektion" und „Manipulation“ menschlichen Lebens, in: Sigrid Graumann, Katrin Grüber (Hrsg.), Medizin, Ethik und Behinderung, Frankfurt a. M. 2003, S. 15-45

Graumann (2003 b): Sigrid Graumann, Sind „Biomedizin“ und „Bioethik“ behindertenfeindlich? Ein Versuch, die Anliegen der Behindertenbewegung für die ethische Diskussion fruchtbar zu machen, Ethik in der Medizin 15 (2003), 3, S. 161-170

Graumann (2006): Sigrid Graumann, Biomedizin und die gesellschaftliche Ausgrenzung von Menschen mit Behinderung, in: Heinrich Greving et al. (Hrsg.), Inklusion statt Integration? Heilpädagogik als Kulturtechnik, Gießen 2006

Haker (2000): Hille Haker, Stellungnahme zur PID. PID Anhörung am 13.11.2000 der Enquetekommission Recht und Ethik der Modernen Medizin des Deutschen Bundestages, Berlin 2000

Hashiloni-Dolev (2007): Yael Hashiloni-Dolev, A Life (Un)Worthy of Living: Reproductive Genetics in Israel and Germany, Dordrecht 2007

Jonas (1979): Hans Jonas, Das Prinzip Verantwortung, Frankfurt a. M. 1979

Judith (2001): Christian Judith, Hättest Du mich abgetrieben? Ein Streitgespräch, Die Zeit, 7 (2001), S. 27 f.

Köbsell (1989): Swantje Köbsell, „Unwertes“ Leben darf abgetrieben werden - Bevölkerungspolitik in der Bundesrepublik, in: Die Grünen im Bundestag, Arbeitskreis Frauenpolitik (Hrsg.): Neue Aspekte in der Abtreibungsdiskussion: Bevölkerungspolitik und Tötungsvorwurf, Bonn 1989

Krones (2006 a): Tanja Krones, The Scope of the Recent Bioethics Debate in Germany: Kant, Crisis and No Confidence in Society, Cambridge Quarterly of Healthcare Ethics 15 (2006), p. 273-281

Krones (2006 b): Tanja Krones, Pränatal- und Präimplantationsdiagnostik aus der Sicht von Paaren mit einem bekannten genetischen Risiko, in: Sigrid Graumann, Katrin Grüber (Hrsg.), Biomedizin im Kontext. Beiträge aus dem Institut Mensch Ethik Wissenschaft, Berlin 2006, S. 201-220

Krones, Richter (2004): Tanja Krones, Gerd Richter, Preimplantation Genetic Diagnosis (PGD): European Perspectives and the German Situation, Journal of Medicine and Philosophy 29 (2004), 5, p. 623-640

Krones et al. (2004): Tanja Krones et al., Einstellungen und Erfahrungen von genetischen Hochrisikopaaren hinsichtlich der Präimplantationsdiagnostik (PID) - Nationale und internationale Ergebnisse, Journal für Reproduktionsmedizin und Endokrinologie, 2 (2004), S. 112-119

Krones et al. (2005): Tanja Krones et al., Public, expert and patients opinions towards preimplantation genetic diagnosis (PGD) in Germany, reproductive Biomedicine online 10 (2005), p. 116-123

Krones et al. (2006): Tanja Krones et al., What is the preimplantation embryo? Social Science and Medicine 63 (2006), p. 1-20

Kuhlmann (2001): Andreas Kuhlmann, Politik des Lebens, Politik des Sterbens. Biomedizin in der liberalen Demokratie, Berlin 2001

Kuhlmann (2003): Andreas Kuhlmann, Therapie als Affront. Zum Konflikt zwischen Behinderten und Medizin, Ethik in der Medizin 15 (2003), p.151-160 


\section{$G$ Behinderungen}

Lindemann-Nelson (2000): James Lindemann-Nelson, Prenatal Diagnosis, Personal Identity, and Disability, Kennedy Institute of Ethics Journal 10 (2000), 3, p. 213-228

Lübbe (2003): Weyma Lübbe, Das Problem der Behindertenselektion bei der pränatalen Diagnostik und der Präimplantationsdiagnostik, Ethik in der Medizin 15 (2003), 3, S. 203-220

Rapp (2000): Rayna Rapp, Testing Women, Testing the Fetus. The Social impact of Amniocentesis in America, New York, London 2000

Rauskolb (2000): Rüdiger Rauskolb, Häufigkeit einer Amniozentese aus Altersgründen - Ein Vergleich zwischen 0st und West, www.nestle-wissdienst.de/html/chefsache/pdf_download/95HLNE3.pdf [15.03.2007]

Rauskolb/Wenzlaff (2004): Rüdiger Rauskolb, Paul Wenzlaff, Amniozentese/Chorionzottenbiopsie aus Altersgründen, www.zq-aekn.de/web_aekn/zqhome. nsf/ContentView/BD3CBADDoCFC8393C1256FDB002BBDAE/ \$Fil/Workshop_4_Amniozentese.pdf

Schindele (1995): Eva Schindele, Schwangerschaft. Zwischen guter Hoffnung und medizinischem Risiko, Hamburg 1995

Schöne-Seifert (1996): Bettina Schöne-Seifert, Medizinethik, in: Julian Nida Rümelin (Hrsg.), Angewandte Ethik. Die Bereichsethiken und ihre theoretische Fundierung, Stuttgart 1996, S. 552-648

Schöne-Seifert/Rippe (1991): Bettina Schöne-Seifert, Klaus-Peter Rippe, Silencing the Singer: Antibioethics in Germany, The Hastings Center Report 21 (1991), 6, p. 20-27

Sermon et al. (2007): Karen D. Sermon et al., ESHRE PGD Consortium data collection VI: cycles from January to December 2003 with pregnancy follow-up to October 2004, Human Reproduction 22 (2007), 2, p. $323-336$

Van den Daele (2003): Wolfgang van den Daele, Empirische Befunde zu den gesellschaftlichen Folgen der Pränataldiagnostik: Vorgeburtliche Selektion und die Auswirkungen auf die Lage Behinderter Menschen. Gentechnologiebericht der Berlin-Brandenburgischen Akademie der Wissenschaften, Berlin 2003

Volz (2003): Sibylle Volz, Diskriminierung von Menschen mit Behinderung im Kontext von Präimplantationsund Pränataldiagnostik, in: Sigrid Graumann, Katrin Grüber (Hrsg.), Medizin, Ethik und Behinderung, Frankfurt a. M. 2003, S. 72-88

Waldschmidt (2003): Anne Waldschmidt, Normierung oder Normalisierung: Behinderte Frauen, der Wille zum „Normkind“ und die Debatte um die Pränataldiagnostik, in: Sigrid Graumann, Ingrid Schneider (Hrsg.), Verkörperte Technik - Entkörperte Frau. Biopolitik und Geschlecht, Frankfurt a. M. 2003, S. 95-109

Wendell (1996): Susan Wendell, The Rejected Body, New York 1996

Wiesemann (2006): Claudia Wiesemann, Von der Verantwortung, ein Kind zu bekommen. Eine Ethik der Elternschaft, München 2006 